\title{
Healthy Baby Second-Hand Smoke Study: project brief
}

\author{
Karen M Emmons, Glorian Sorensen, Neil Klar, Lisa Digianni, Gillian Barclay, \\ Kaydee Schmidt, S Katharine Hammond
}

Although a number of studies have assessed differences in personal and interpersonal factors between smokers and non-smokers (for example, age, smoking during previous pregnancies, partner smoking status), there has been little attention to the social context in which women make decisions about prenatal health behaviours. ${ }^{1}$ For pregnant women who are poor, undereducated, and facing housing or economic crises, the immediate benefits provided by smoking may outweigh the long term risks of smoking during pregnancy. In order to improve the effectiveness of smoking interventions for pregnant women, it may be necessary to place smoking in the broader social context in which these women live, and integrate it into other programs that address basic life needs.

The Healthy Baby Second-Hand Smoke Study was a pilot program designed to evaluate the role of public health nurses in delivering a novel smoking intervention to limited income, high risk pregnant women. The Healthy Baby Program (HBP) is a social service program that provides medical and social service outreach to low income pregnant women who are at risk for poor birth outcomes because of their medical history or socioeconomic circumstances. A collaboration was formed with the HBP to develop the HBP Second-Hand Smoke intervention, which was designed to motivate pregnant women towards reducing their household exposure to second hand smoke and towards smoking cessation, and to provide skills to those who want to quit smoking. Because of the attention to other life issues provided by the Healthy Baby Program, it was hypothesised that participants might be more able to address smoking as an important health issue.

Dana-Farber Cancer Institute, Boston

N Klar

L Digianni

Boston Public Health Commission, Boston K Schmidt

University of California at Berkeley, Berkeley, California, USA

S K Hammond

Correspondence to: Karen M Emmons, PhD, Harvard School of Public Health and the Dana-Farber Cancer Institute, Division of Cancer Institute, Division of
Community-Based Research, 44 Binney Street, Boston

MA 02115USA;

karen_emmons@

karen_emmons@

\section{Methods}

This study was conducted with clients served by the Healthy Baby Program (HBP). The HBP team includes nurses and advocates who serve Boston neighbourhoods with the highest rates of infant deaths and low birth weight. Nursing services include health education, medical monitoring, referrals, and follow up; the advocates focus on social needs of the clients and providing linkages to community services. The program received referrals through neighbourhood health centres and a mobile family van. Women are eligible to receive services from HBP if they are pregnant and/or within three months postpartum, are at risk for poor pregnancy outcome or low birth weight, or are at risk for pregnancy complications.
A quasi-experimental historical comparison design was used because of concerns about contamination across groups. The first 12 months of the study period comprised the "usual care" (UC) phase; following completion of this phase, the nurses were trained in motivational interviewing and the study protocol, and the "motivational intervention" (MI) phase of the study began. During the entire study period, the smoking status of all clients served by the HBP was assessed; any HBP participant who was a current smoker or recent quitter was eligible for this study. Seven day point prevalence abstinence was used as the primary outcome measure.

Participants in the HBP who expressed interest provided permission for the study team to contact them; women who agreed to participate received a home visit from a research assistant during which informed consent was provided. Study assessments were conducted at baseline, six week prenatal follow up, and at one month postpartum. Household nicotine levels were measured at each assessment using passive sampling dosimetry ${ }^{2}$; a saliva sample was also collected in order to assess the mother's cotinine concentration.

During the usual care phase of the study, participants completed the study assessments. Usual care participants received the standard smoking cessation intervention provided by the Healthy Baby Program, which consisted of a strong recommendation to quit smoking for the sake of the baby's health.

The study intervention was provided by Healthy Baby nurses, and was incorporated into their regular home visits to program participants. The study intervention utilised a motivational interviewing approach, focused on reduction of second hand smoke and smoking cessation. ${ }^{3}$ The intervention included feedback about the nicotine levels found in the participant's home. ${ }^{5}$ Following the initial home visit at which feedback about nicotine levels was provided, participants were asked to try different strategies for reducing household second hand smoke levels, and then received feedback on the nicotine results following the prenatal follow up assessment. Smoking was discussed at all subsequent HBP home visits.

\section{Results}

Sixty two women were recruited for the UC group, and 52 women for the MI group. Participant characteristics are listed in table 1. There was a trend for participants in the UC group to be younger than those in the $\mathrm{MI}$ group, and significantly fewer of the UC 
Table 1 Demographic and socioeconomic characteristics of study participants

\begin{tabular}{|c|c|c|c|}
\hline Participants & UC group & MI group & $p$ Value \\
\hline Age (years) (mean (SD)) & $23.4(5.6)$ & $25.8(6.6)$ & 0.05 \\
\hline Race & & & NS \\
\hline Black/African America & $62.3 \%$ & $53.8 \%$ & \\
\hline White, not hispanic & $13.1 \%$ & $17.3 \%$ & \\
\hline Other & $24.6 \%$ & $28.8 \%$ & \\
\hline Hispanic & $24.6 \%$ & $26.9 \%$ & \\
\hline Number of children & & & NS \\
\hline 1 & $29.3 \%$ & $33.0 \%$ & \\
\hline $2+$ & $25.9 \%$ & $11.8 \%$ & \\
\hline Education & & & NS \\
\hline$<$ High school & $55.1 \%$ & $44.2 \%$ & \\
\hline High school & $25.9 \%$ & $30.8 \%$ & \\
\hline$>$ High school & $19.0 \%$ & $25.0 \%$ & \\
\hline Marital status & & & 0.01 \\
\hline Married/living as married & $12.1 \%$ & $32.7 \%$ & \\
\hline Single/divorced, widowed & $87.9 \%$ & $67.3 \%$ & \\
\hline On Medicaid (\%) & $70.2 \%$ & $72.5 \%$ & NS \\
\hline Smoking status & & & 0.05 \\
\hline Current smoker & $81.7 \%$ & $94.2 \%$ & \\
\hline Recent quitter & $18.3 \%$ & $5.8 \%$ & \\
\hline Cigarettes smoked/day (mean (SD)) & $8.1(5.3)$ & $8.4(8.5)$ & NS \\
\hline Nicotine dependent & $40.3 \%$ & $52.0 \%$ & NS \\
\hline Stage of readiness to quit & & & NS \\
\hline Precontemplation & $32.6 \%$ & $31.9 \%$ & \\
\hline Contemplation & $16.3 \%$ & $27.7 \%$ & \\
\hline Preparation & $27.9 \%$ & $34.0 \%$ & \\
\hline Action & $23.3 \%$ & $6.4 \%$ & \\
\hline Some/most of family/friends smoke & $72.4 \%$ & $73.0 \%$ & NS \\
\hline
\end{tabular}

UC, usual care; MI, motivational intervention; NS, not significant.

Table 2 Study outcomes

\begin{tabular}{|c|c|c|c|c|c|c|}
\hline \multirow[b]{2}{*}{ Variable } & \multicolumn{3}{|c|}{ Prenatal follow up } & \multicolumn{3}{|c|}{ Postpartum follow up } \\
\hline & $U C$ & $M I$ & $p$ Value & $U C$ & $M I$ & $p$ Value \\
\hline \multicolumn{7}{|l|}{ Recent quitters at baseline } \\
\hline$\%$ relapsed $(n=17)$ & 10.0 & 0.0 & NS & 22.2 & 50.0 & NS \\
\hline Smokers at baseline $(n=96)$ & & & & & & \\
\hline$\%$ abstinent & 5.6 & 6.7 & NS & 0.0 & 0.0 & NS \\
\hline \multicolumn{7}{|l|}{ Smoking rate } \\
\hline$<5$ cigarettes/day $(\%)$ & 36.1 & 38.5 & NS & 21.9 & 25.9 & NS \\
\hline $5+$ cigarettes/day $(\%)$ & 63.9 & 61.5 & & 78.1 & 74.1 & \\
\hline Stage of readiness to quit & & & NS & & & 0.01 \\
\hline Precontemplation & 41.2 & 58.3 & & 42.9 & 73.9 & \\
\hline Contemplation & 23.5 & 25.0 & & 46.4 & 8.7 & \\
\hline Preparation & 32.4 & 16.7 & & 10.7 & 17.4 & \\
\hline Action & 2.9 & 0.0 & & 0.0 & 0.0 & \\
\hline
\end{tabular}

participants were married. There was also a greater number of recent quitters in the UC intervention; there were no other differences between study groups.

The study outcomes are presented in table 2 . Analyses were adjusted for baseline differences between groups, and intention to treat analyses were used. There were no significant differences in cessation rates between the two groups. In addition, no differences were found in follow up smoking rate. Although overall attrition rates were reasonable given the socioeconomic status and mobility of this population ( $35 \%$ by postpartum), there was a higher attrition rate in the intervention group ( $48 \%$ in intervention $v 30 \%$ in control), which further reduced our power to evaluate study outcomes.

\section{Discussion}

The motivational intervention was not found to increase smoking cessation rates beyond the levels found in the usual care group. Although we adjusted for baseline differences in the analyses, it is important to note that there were differences in key demographic characteristics related to smoking between the two groups. Significantly more participants in the UC group were recent quitters, which is a predictor of postnatal smoking status. In addition, a larger percentage of women in the MI group were nicotine dependent; although this difference did not reach significance, it is worthy of note and may have made it more difficult for women in the MI group to consider quitting.

There were a number of important lessons learned in this pilot study. First, recruitment of very low income smokers to a smoking intervention was very challenging. Because of the structure of the intervention, we had to rely on HBP staff to recruit their clients to the study. Although HBP staff received a substantial amount of training on recruitment and on refocusing recruitment efforts away from smoking cessation, the study was often represented as a smoking cessation intervention, which likely affected participation rates. Second, the intervention dose delivered was less than planned. The HBP nurses reported that they face a number of challenges in working with their clients, including difficulty contacting them because of lack of working phones, and difficulty tracking them owing to frequent and often unexpected moves. Resources were not available to increase the number of contact attempts in order to deliver the smoking intervention, and thus the intervention dose delivered was less than optimal. Third, the time available to train the Healthy Baby Program nurses in the motivational intervention was limited. Nurses were initially trained in motivational interviewing in an 8 hour training session, with three, 2 hour follow up training sessions. In addition, follow up consultation was provided on an individual basis. However, during the individual follow up consultation it was clear that more intensive training would have been helpful, and that many program staff found it difficult to utilise a motivational style. One solution to this challenge would be to select nurses within a program who have good counselling skills, particularly related to motivational enhancement, and an interest in smoking, and select these nurses as "champions" who would deliver the intervention to all eligible pregnant women.

It is often challenging for outside research groups to gain trust with community based service programs and the community itself. It is essential that researchers develop their relationships with the community over an extended period of time; however, the requirements of a two year research project such as this greatly limited the amount of relationship development that could occur outside of the research needs, and thus may have impacted on the acceptance and delivery of the intervention. This is a challenge that researchers and funders alike need to address.

Overall, patients reported being quite satisfied with the intervention. In addition, the providers enjoyed having the opportunity to learn new skills, and found the motivational interviewing approach to be useful for working with clients on smoking as well as other behaviours. Although it is disappointing that the motivational intervention did not impact on 
smoking cessation rates among study participants, the findings of this pilot study suggest that perhaps more intensive interventions, with more extensive training of delivery staff, are needed to target smoking among very low income populations. The results of this study should not be interpreted as an indictment of a motivational intervention model, but rather as a call to conduct more extensive research into the underlying contextual factors that may influence smoking among low income pregnant women, and an effort to develop new intervention models that target these factors.
This research is supported in part by the Robert Wood Johnson Foundation, and by Liberty Mutual Insurance Company, Aetna, The Boston Company, and NYNEX.

1 Emmons KM. Health behaviors in a social context. In: Berkman L, Kawachi I, eds. Social epidemiology. Oxford Press, 2000: 242-66.

2 Hammond SK. Evaluating exposure to environmental tobacco smoke. Boca Raton: Lewis Publishers, 1993.

3 Miller W, Rollnick S. Motivational interviewing: preparing people to change addictive behaviors. New York: Guilford Press, 1991.

4 Rollnick S, Miller W. What is motivational interviewing? Behavioural \& Cognitive Psychotherapy 1995;23:325-34.

5 Hovell M, Meltzer S, Zakarian J, et al. Reduction of environmental tobacco smoke exposure among asthmatic children: a controlled trial. Chest 1994;106:440-6.

\title{
The Kaiser Permanente prenatal smoking cessation trial: when more isn't better, what is enough?
}

\author{
Daniel H Ershoff, Virginia P Quinn, Neal R Boyd, Julie Stern, Margaret Gregory, \\ David Wirtschafter
}

The effectiveness of low cost smoking interventions targeted to pregnant women has been demonstrated, although few gains in absolute cessation rates have been reported in the past decade. Under conditions of typical clinical practice, this study examined whether outcomes achieved with brief counselling from prenatal care providers and a self help booklet could be improved by adding more resource intensive cognitive behavioural programs.

\section{Design}

Randomised clinical trial.

\section{Setting}

A large group model manage care organisation.

Kaiser Permanente Southern California Group, Pasadena, California, USA

D H Ershoff

V P Quinn

J Stern

M Gregory

D Wirtschafter

Fox Chase

Comprehensive

Cancer Center

N R Boyd

Correspondence to: Dr VP Quinn, Southern California Permanente

Medical Group, Department

of Research and Evaluation,

Walnut Center 911,

Pasadena, CA 91188, USA;

Virgina.P.Quinn@kp.org

\section{Participants}

Three hundred and ninety English speaking women 18 years of age or older who self reported to be active smokers at their initial prenatal appointment.

\section{Intervention}

Participants were randomised to one of three groups: (1) a self help booklet tailored to smoking patterns, stage of change, and lifestyle of pregnant smokers; (2) the booklet plus access to a computerised telephone cessation program based on interactive voice response technology; or (3) the booklet plus proactive telephone counselling from nurse educators using motivational interviewing techniques and strategies. No attempt was made to change smoking related usual care advice from prenatal providers.

\section{Main outcome measure}

Biochemically confirmed abstinence measured by concentration of cotinine in urine samples obtained during a routine prenatal visit at approximately the 34 th week of pregnancy.

\section{Results}

Twenty per cent of participants were confirmed as abstinent with no significant differences found between intervention groups. Multivariate baseline predictors of cessation included number of cigarettes smoked per day, confidence in ability to quit, exposure to passive smoke, and education level. No differential intervention effects were found within strata of these predictors or by baseline stage of readiness to change. Cessation rates among heavier smokers were strikingly low in all intervention groups.

\section{Conclusion}

Neither a computerised telephone cessation program nor systematic provision of motivational counselling improved cessation rates over a tailored self help booklet delivered within the context of brief advice from prenatal providers. Innovative strategies need to be developed to increase the effectiveness of existing prenatal smoking interventions. Special attention should be paid to the needs of heavier smokers.

First published as an abstract to an article in $A m \mathcal{F}$ Prev Med 1999;17:161-8. 